\title{
The Lascoux, Leclerc and Thibon algorithm and Soergel's tilting algorithm
}

\author{
Steen Ryom-Hansen
}

Received: February 5, 2004 / Revised: May 3, 2005 / Accepted: May 19, 2005

(C) Springer Science + Business Media, Inc. 2006

\begin{abstract}
We generalize Soergel's tilting algorithm to singular weights and deduce from this the validity of the Lascoux-Leclerc-Thibon conjecture on the connection between the canonical basis of the basic submodule of the Fock module and the representation theory of the Hecke-algebras at root of unity.
\end{abstract}

Keywords Tilting modules $\cdot$ Crystal basis $\cdot$ Fock module $\cdot$ Kashdan-Lusztig polynomials

\section{Introduction}

In this paper we show that the Lascoux-Leclerc-Thibon conjecture [9] on the connection between the canonical basis of the basic submodule of the Fock module and the representation theory of Hecke-algebras at a root of unity follows from the truth of Soergel's tilting algorithm. This result was independently obtained by Goodman and Wenzl [6] and has also been proved by Leclerc-Thibon [10].

Our proof (which has existed in various versions since 1997) differs in several ways from the above proofs, first of all it relies notationally as wells as philosofically directly on the principle of graded representation theory as exposed in the paper of Andersen, Jantzen and Soergel [3]. Indeed, as our first result we explain how the AJS-formalism naturally leads to an extension of Soergel's algorithm so as to be able to deal with singular weights, i.e. weights lying on several reflecting hyperplanes. This singular combinatorics is important from our point of view, since the partitions appearing in the LLT-algorithm typically correspond to very singular weights. However, it should be noted that since the basic setting of [3] is that

Supported in part by Programa Reticulados y Ecuaciones and by FONDECYT grant 1051024.

S. Ryom-Hansen $(\bowtie)$

Instituto de Matemática y Física, Univerdad de Talca, Chile

e-mail: STEEN@INSTMAT.UTALCA.CL 
of Frobenius kernels, we cannot formally use the results of that paper. Indeed, we only check in type A that the singular combinatorics does not depend on the path of weights chosen and do so by comparing it with the LLT algorithm for large values of $l$, the order of the root of unity.

We then go on to show that our singular combinatorics yields the correct tilting characters using the correctness of the original Soergel algorithm together with some known properties of tilting modules and translation functors. Finally, we show that the LLT-algorithm is a special case of our singular combinatorics; this involves a detailed analysis of the correspondence between partitions and weights.

I wish to thank B. Leclerc and W. Soergel for useful discussions.

\section{Preliminaries}

In this section we shall setup the notation needed. Let $\mathfrak{g}$ be a finite dimensional semisimple Lie-algebra over the complex numbers and let $U_{q}(\mathfrak{g})$ be the associated quantum group at an $l$-th root of unity, see e.g. [1] for the precise definition. The representation theory of $U_{q}(\mathfrak{g})$ is labeled by the set of dominant weights $P^{+}$and the blocks correspond under this labeling to orbits in $P^{+}$under the affine Weyl group $W_{l}$. Thus, for every weight $\lambda \in P^{+}$there is a standard module $\Delta(\lambda)$, a costandard module $\nabla(\lambda)$, a simple module $L(\lambda)$ and a tilting module $Q(\lambda)$. See $[1,3,12]$ for more details.

We shall make extensive use of the following notation on alcove geometry introduced in [3]. Let $\Omega$ be a regular orbit of $W_{l}$ in $P^{+}$, i.e. one consisting of regular weights (lying on no walls), and let $\Gamma$ be a singular orbit. Following [3], for $\lambda \in \Omega$ we denote by $\lambda_{\Gamma}$ the unique element of $\Gamma$ in the closure of the alcove of $\lambda$. Furthermore, we set

$$
\begin{aligned}
& u(\lambda, \Gamma)=\left|\left\{H \in \mathcal{H} \mid \lambda_{\Gamma} \in H, \lambda<H\right\}\right| \\
& o(\lambda, \Gamma)=\left|\left\{H \in \mathcal{H} \mid \lambda_{\Gamma} \in H, \lambda>H\right\}\right|
\end{aligned}
$$

where $\mathcal{H}$ denotes the set of reflecting hyperplanes for $W_{l}$. Using this we can define $\Omega_{s}$ by

$$
\Omega_{s}:=\{\lambda \in \Omega \mid u(\lambda, \Gamma)=0\}
$$

Let us illustrate this on an A2 example:

\section{Example 1.}

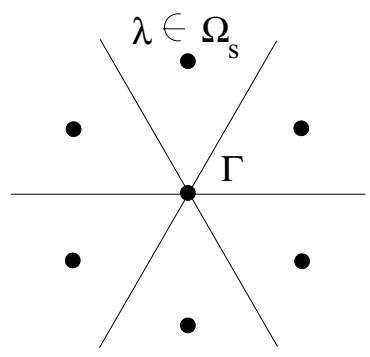


Let us briefly recall Soergel's algorithm as well as the LLT-algorithm. Let $\mathcal{A}$ be the set of alcoves, $\mathcal{A}^{+}$the set of dominant alcoves. Then the Grothendieck group of $\Omega$ can be identified with $\mathbb{Z}\left[\mathcal{A}^{+}\right]$. Soergel's algorithm produces for each $A \in \mathcal{A}^{+}$an "indecomposable pattern" $P_{A} \in \mathbb{Z}[q]\left[\mathcal{A}^{+}\right]$, by which we mean an element $P_{A} \in \mathbb{Z}[q]\left[\mathcal{A}^{+}\right]$on the form

$$
P_{A}(q)=A+\sum_{B<A} P_{A B}(q)
$$

where $P_{A B}(q) \in q \mathbb{Z}[q]$. This pattern contains information about the character of the tilting module $Q(A)$ with highest weight $\lambda$ where $\lambda \in A \cap \Omega$. In formulas:

$$
[Q(A), \Delta(B)]=P_{A B}(1)
$$

where $\Delta(B)$ is shorthand for $\Delta(\mu)$, where $\mu \in B \cap \Omega$. The procedure for calculating $P_{A}$ is a recursion on $\mathcal{A}$. It involves for each wall $s$ of the fundamental alcove an operator $\Theta_{s}$ on $\mathbb{Z}[q]\left[\mathcal{A}^{+}\right]$taking the indecomposable pattern to a sum of indecomposable patterns (i.e. patterns with more than one coefficient having a constant term). This operator is defined through the formula

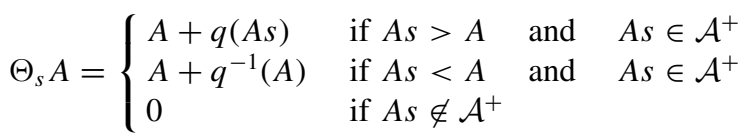

and linearity; $A s$ is here the mirror alcove of $A$ under the reflection given by $s$. The following picture illustrates the first two cases of this action in an alcove geometry of a type A situation, where the reflection is going upwards in the first case, downwards in the second case.

\section{Example 2.}
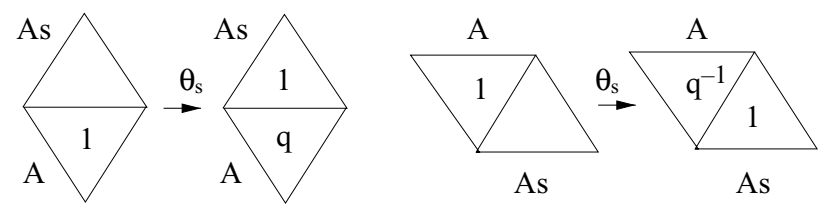

One then subtracts inductively known indecomposable patterns to arrive at the new indecomposable pattern, whose top alcove is the only one with a coefficient involving a constant term. We illustrate the algorithm on the following A3 examples, and refer to [12] for more details. 


\section{Example 3.}
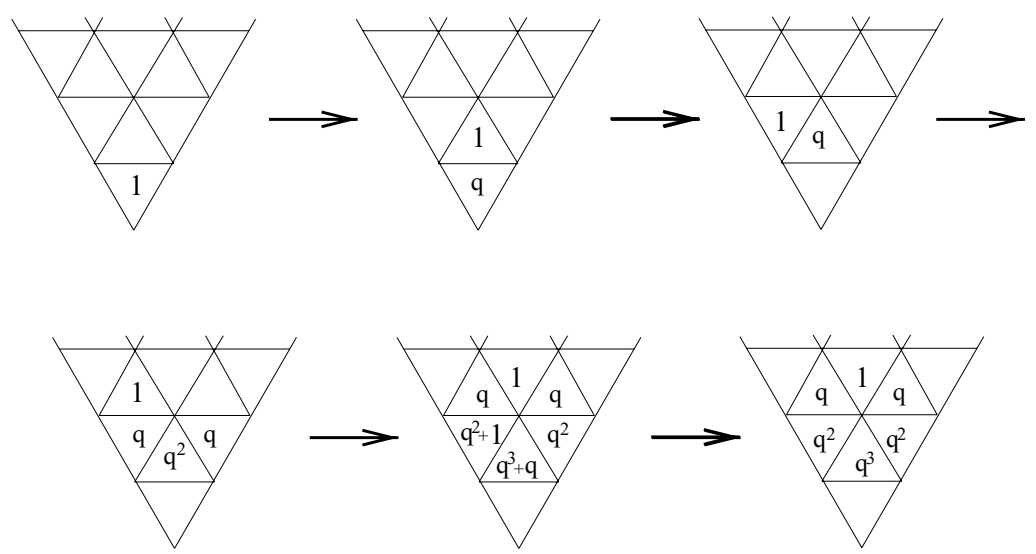

Following the terminology introduced in [12], we shall denote the above algorithm a "combinatorics" for tilting modules.

We now briefly recall the LLT-algorithm. Let $\mathcal{F}_{q}=\bigoplus_{\lambda \in \operatorname{Par}} \mathbb{Q}(q)|\lambda\rangle$ be the $q$-Fock space with basis parameterized by the set of all partitions Par. It can be made into an integrable module for $U_{q}\left(\widehat{\mathfrak{s l}}_{l}\right)$ and thus has a crystal basis. The LLT-algorithm calculates the global basis of the basic submodule $M$ of $\mathcal{F}_{q}$, which is the one generated by the empty partition. Let $L$ be the $\mathbb{Z}[q]$-sublattice of $\mathcal{F}_{q}$ with basis $\{|\lambda\rangle \mid \lambda \in \operatorname{Par}\}$. The lower global basis element $G(\lambda)$ of $M$ is characterized by the following conditions

$$
\overline{G(\lambda)}=G(\lambda), \quad G(\lambda)=|\lambda\rangle \bmod q L
$$

for $\lambda \in \operatorname{Par}_{l}$, i.e. an $l$-regular partition, where ${ }^{-}$is the involution of $M$ given by

$$
\bar{\emptyset}=\emptyset, \quad \overline{f_{i} w}=f_{i} \bar{w} \quad \forall i \text { and } \bar{q}=q^{-1},
$$

Let $d_{\lambda \mu}(q)$ be defined by

$$
G(\lambda)=\sum_{\mu} d_{\lambda \mu}(q)|\mu\rangle
$$

Then $d_{\lambda \mu}(q) \in \mathbb{Z}[q], d_{\lambda, \mu}(q)=0$ unless $\lambda \unlhd \mu$ and $d_{\lambda, \lambda}(q)=1$. Call an element $w$ of $M$ selfdual if it satisfies $\bar{w}=w$. A selfdual element $w$ can be written in the form

$$
w=\sum_{\lambda} a_{\lambda}(q) G(\lambda)
$$

for some $a_{\lambda}(q) \in \mathbb{Z}\left[q, q^{-1}\right]$ satisfying $\overline{a_{\lambda}(q)}=a_{\lambda}(q)$. The LLT-algorithm first constructs for each regular partition $\lambda$ a selfdual element $w_{\lambda}$ such that the coefficient of $G(\lambda)$ in $w_{\lambda}$ is 1 and such that $\mu<\lambda$ for all other occurring $G(\mu)$. From this, $G(\lambda)$ is obtained by linear algebra.

LLT conjectured that for $\lambda$ an $l$-regular partition

$$
d_{\lambda \mu}(1)=[S(\mu), D(\lambda)]
$$


where $S(\mu)$ and $D(\lambda)$ are the Specht and the simple modules for the Hecke algebra of type A specialized at an l'th root of unity. This conjecture was first proved by Ariki [2] using the geometric approach to the crystal/canonical basis. The goal of this paper, however, is to demonstrate that it also follows from Soergel's algorithm.

\section{Singular tilting modules}

We first need to generalize some results of Andersen on singular tilting modules.

Let $\Omega$ be a regular $W_{l}$-orbit in $P^{+}$containing $\lambda$ and let $\Gamma$ be a singular orbit containing $\mu$. Let $T_{\Gamma}^{\Omega}$ be the Jantzen translation functor from the $\Gamma$-block to the $\Omega$-block, see e.g. [1]. We then have the following proposition

Proposition 1. Assume $\lambda_{\Gamma}=\mu$ and $\lambda \in \Omega_{s}$. Then

$$
T_{\Gamma}^{\Omega} Q(\mu) \cong Q(\lambda)
$$

Proof: One can copy the proof of Proposition 5.6 in Andersen's paper [1]. In that paper $\Gamma$ is assumed semiregular; however the proof carries over to our situation.

As a corollary, we obtain that the character of the singular tilting modules can be calculated from the regular ones:

Corollary 1. Let $\mu, \bar{\mu} \in \Gamma$. Then

$$
\left[Q(\mu), \Delta(\bar{\mu}]=\frac{1}{N_{\Gamma}} \sum_{\bar{\lambda}: \bar{\lambda}_{\Gamma}=\bar{\mu}}[Q(\lambda), \Delta(\bar{\lambda})]\right.
$$

where $\lambda \in \Omega_{s}$ with $\lambda_{\Gamma}=\mu$. (As in [AJS] $N_{\lambda}$ denotes the number of hyperplanes in $\mathcal{H}$ such that $\lambda \in H)$.

Proof: We know that

$$
T_{\Omega}^{\Gamma} T_{\Gamma}^{\Omega} Q(\mu) \cong Q(\mu)^{\oplus N_{\Gamma}} .
$$

Hence we obtain the Corollary from the Theorem using the standard properties of translation functors.

\section{A combinatorics of graded translation functors}

We saw in the previous section that the singular tilting characters can be deduced from the regular ones. Now there may be no regular weights in the weight lattice, so we still insist on constructing a combinatorics of graded translation functors that works in the singular case. This section is devoted to that task. We do it by assuming the existence of a formalism of graded translation functors for our $U_{q}(\mathfrak{g})$-representation theory having the same formal 
properties as the ones in [3] for the Frobenius kernels. We then show that this naturally leads to a combinatorics for singular tilting modules.

Let thus $\Omega_{\tau}=\Omega, \Gamma_{\lambda}=\Gamma$ and $\Pi_{\mu}=\Pi$ be the orbits under $W_{l}$ of $\tau, \lambda$ and $\mu$. Assume furthermore $\tau$ regular and $\mu$ more singular than $\lambda$, i.e. $W_{\lambda} \subseteq W_{\mu}$.

\section{Example 4.}

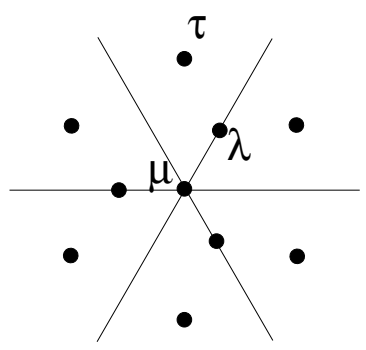

Let $\mathcal{K}(\tau), \mathcal{K}(\lambda)$ and $\mathcal{K}(\mu)$ be the corresponding $\mathbb{Z}$-graded Grothendieck groups, i.e. $\mathcal{K}(\tau)=$ $\mathbb{Z}[q][\Omega]$ etc. As mentioned above they so far have been constructed only in the case of Frobenius kernels. According to the [3]-philosophy, they should come with a system of operators (graded translation functors)

$$
\begin{array}{lll}
T_{\mu, \lambda}^{*}: \mathcal{K}(\mu) \rightarrow \mathcal{K}(\lambda), & T_{\lambda, \tau}^{*}: \mathcal{K}(\lambda) \rightarrow \mathcal{K}(\tau), & T_{\mu, \tau}^{*}: \mathcal{K}(\mu) \rightarrow \mathcal{K}(\tau) \\
T_{*}^{\lambda, \mu}: \mathcal{K}(\lambda) \rightarrow \mathcal{K}(\mu), & T_{*}^{\tau, \lambda}: \mathcal{K}(\tau) \rightarrow \mathcal{K}(\lambda), & T_{*}^{\tau, \mu}: \mathcal{K}(\tau) \rightarrow \mathcal{K}(\mu)
\end{array}
$$

as well as a system of $T^{!}$and $T_{!}$operators and a duality $D$ relating the operators as follows

$$
D \circ T_{\mu, \lambda}^{*} \circ D=T_{\mu, \lambda}^{!}
$$

etc. Furthermore the duality should anticommute with the $\mathbb{Z}$-shift in the categories, i.e. $D \circ\langle 1\rangle=\langle-1\rangle \circ D$.

Now [3] p. 253 suggests that $T_{\mu, \tau}^{*}$ and $T_{*}^{\tau, \mu}$ should satisfy the following rules:

$$
\begin{aligned}
& T_{*}^{\tau, \mu} \Delta(\tau)=\Delta\left(\tau_{\Pi}\right)\langle o(\tau, \Pi)\rangle \\
& T_{\mu, \tau}^{*} \Delta(\mu)=\sum_{\tau: \tau_{\Pi}=\mu} \Delta(\tau)\langle o(\tau, \Pi)\rangle
\end{aligned}
$$

and similarly for $T_{\lambda, \tau}^{*}$ and $T_{*}^{\tau, \lambda}$. We take this as our definition.

But then the transitivity forces us to define $T_{*}^{\lambda, \mu}$ and $T_{\mu, \lambda}^{*}$ by

$$
\begin{aligned}
& T_{*}^{\lambda, \mu} \Delta(\lambda)=\Delta\left(\lambda_{\Pi}\right)\langle o(\lambda, \Pi)\rangle \\
& T_{\mu, \lambda}^{*} \Delta(\mu)=\sum_{\lambda: \lambda_{\Pi}=\mu} \Delta(\lambda)\langle o(\lambda, \Pi)\rangle
\end{aligned}
$$


i.e. the very same formulas as translation to and from the regular orbits. One should here notice that the expression $\langle o(\lambda, \Pi)\rangle$ makes sense for all weights and that

$$
o\left(\tau, \Omega_{\lambda}\right)+o\left(\tau_{\Gamma_{\lambda}}, \Pi_{\mu}\right)=o\left(\tau, \Pi_{\mu}\right)
$$

Now $T_{*}^{\lambda, \mu}=T_{!}^{\lambda, \mu}$; hence $T_{*}^{\lambda, \mu}$ should preserve selfduality (i.e should commute with $D$ ). On the other hand we have that $T_{\mu, \lambda}^{!}=T_{\mu, \lambda}^{*}\left\langle-2\left(N_{\mu}-N_{\lambda}\right)\right\rangle$, so the operator that preserves selfduality should be

$$
T_{\mu, \lambda}^{*}\left\langle N_{\lambda}-N_{\mu}\right\rangle
$$

Using the convention that $\Delta(\lambda)=0$ whenever $\lambda \notin P^{+}$and that

$$
o(\lambda, \Pi)+u(\lambda, \Pi)=N_{\mu}-N_{\lambda}
$$

we arrive at the following first step for our combinatorics for tilting modules. We first assume that the character of $Q(v)$ for $v \in \mathcal{K}(\lambda)$ comes from an "indecomposable pattern" i.e. an element of $\mathbb{Z}[q] \mathcal{K}(\lambda)$ on the form

$$
v+\sum_{v^{\prime}<v} P_{v^{\prime}, v}(q) v^{\prime}
$$

with $P_{v^{\prime}, v}(q) \in q \mathbb{Z}[q]$, We then assume that there are operators akin to the $\Theta_{s}$ of the Section 2. The above considerations lead us to choosing these as follows.

Definition 1. Singular combinatorics for tilting modules: Step 1:

Let $\lambda$ and $\mu$ be as above, i.e. with $\mu$ more singular than $\lambda$ and let $\mathcal{K}(\mu), \mathcal{K}(\lambda)$ be the corresponding graded categories. Then the graded translation functors $\Theta^{*}$ and $\Theta_{*}$ that take tilting modules to tilting modules, are:

$$
\begin{aligned}
& \Theta_{*}: \mathcal{K}(\lambda) \rightarrow \mathcal{K}(\mu): \Delta(\lambda) \mapsto \Delta\left(\lambda_{\Pi}\right)\langle o(\lambda, \Pi)\rangle \\
& \Theta^{*}: \mathcal{K}(\mu) \rightarrow \mathcal{K}(\lambda): \Delta(\mu) \mapsto \sum_{\lambda: \lambda_{\Pi}=\mu} \Delta(\lambda)\langle-u(\lambda, \Pi)\rangle
\end{aligned}
$$

Let us illustrate this definition on an example

Example 5.

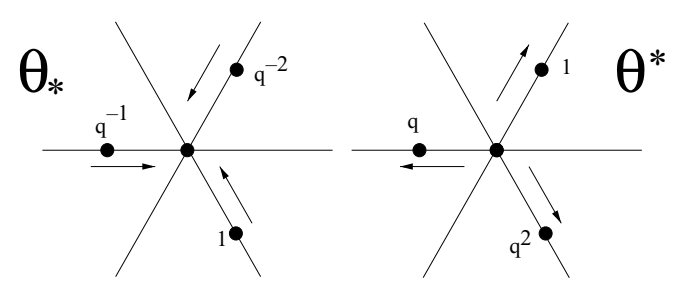


We here used the convention that the shift $\langle 1\rangle$ in the graded category $\mathcal{K}(\lambda)$ corresponds to the multiplication by $q^{-1}$.

The next step of our combinatorics is to explain how to obtain the indecomposable pattern with highest weight $\mu$.

This is, like in the regular case, an inductive procedure, starting with the weights $v$ of the fundamental alcove, for which the pattern $P_{v}(q)$ equals $v$ itself. We then work ourselves upwards through the weight lattice with successive functors $\Theta^{*}$ and $\Theta_{*}$ always trying to produce indecomposable patterns.

If $P(\mu)$ is an indecomposable pattern in $\mathcal{K}(\mu)$ then it is clear from the definition that $\Theta^{*} P(\mu)$ will remain indecomposable.

Now applying $\Theta_{*}$ to an indecomposable pattern $P(\lambda)$ in $\mathcal{K}(\lambda)$ will generally not produce an indecomposable pattern—and $\Theta_{*} P(\lambda)$ will generally not even have coefficients in $\mathbb{Z}[q]$. We can therefore not just mimic Soergel's procedure of subtracting inductively known patterns to arrive at something indecomposable.

On the other hand the coefficient of the leading (maximal) weight $\mu$ will be " 1 " since $\Theta_{*}$ does not lower the $q$-power when going upwards. For each occurrence in the arising pattern of a $q^{i} v$ with $i$ negative or zero, we then subtract $\gamma(q) P_{\mu}(q)$ where $\gamma(q) \in \mathbb{Z}\left[q, q^{-1}\right]$ satisfies $\gamma(q)=\gamma\left(q^{-1}\right)$. Repeating this eventually produces an indecomposable pattern with leading coefficient " 1 ".

Since there may be no weights inside the alcoves we need to generalize the previous considerations slightly to know what happens when translating between arbitrary singular blocks. Since translation functors should depend only on the alcove geometry, we may pretend that there exist regular weights. Then one finds for any $\lambda, \lambda^{\prime} \in P^{+}$a $\mu$ such that

$$
W_{\lambda} \supseteq W_{\mu}, \quad W_{\lambda^{\prime}} \supseteq W_{\mu}, \quad W_{\mu} \supseteq W_{\lambda} \cap W_{\lambda^{\prime}}
$$

Let us illustrate this situation on an example

\section{Example 6.}

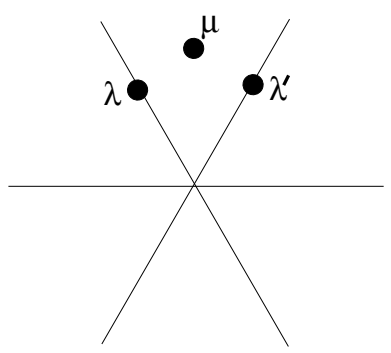

Now for the ordinary translation functors we have transitivity in that case: $T_{\lambda}^{\lambda^{\prime}}=T_{\mu}^{\lambda^{\prime}} \circ T_{\lambda}^{\mu}$. We can therefore take the composite $\Theta_{*} \circ \Theta^{*}$ as the graded version of $T_{\lambda}^{\lambda^{\prime}}$. 


\section{Example 7.}

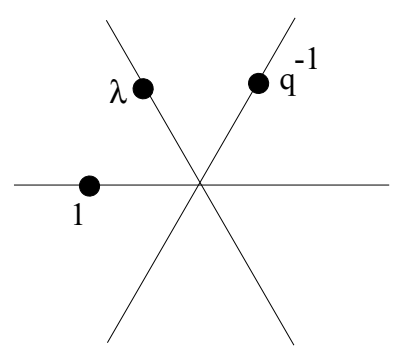

Finally, we kill all weights which are not in the dominant Weyl chamber; this is analogous to Soergel's algorithm. Let us formulate all of this in one statement

Definition 2. Singular combinatorics, step two. Given $\lambda \in P^{+}$. Let $v_{1}, v_{2}, \ldots v_{N}$ be the set of weights in $P^{+}$strictly less than $\lambda$ in the usual order and assume inductively given tilting patterns $P_{v_{i}}(q) \in \mathbb{Z}[q] \mathcal{A}$ for each $v_{i}$. Let $v$ be a $v_{i}$ in the closure of the alcove of $\lambda$. Perform the relevant functor $\Theta_{*}$ or $\Theta^{*}$ or composite thereof on $P_{v}(q)$ and subtract appropriate $P_{v_{i}}(q)$ 's as described to arrive at an indecomposable pattern. This is $P_{\lambda}(q)$.

\section{Comparing the combinatorics}

We now have a singular alcove combinatorics. It is clear that it gives Soergel's combinatorics if we only use semiregular orbits and translate through the walls. We must check that it always leads to the same answer, independently of the chosen path of weights. Once this has been established, the algorithm will be correct, since we can choose a path

$$
v_{1}, v_{2}, v_{3}, \ldots v_{N}, v_{N+1}, \ldots v_{N+K}
$$

such that $v_{1}, v_{2}, v_{3}, \ldots v_{N}$ are regular and semiregular while $v_{N+1}, \ldots v_{N+K}$ have increasing stabilizers. And for such a path, our algorithm yields the correct answer, by the correctness of Soergel's algorithm together with Corollary 1 and the construction of $\Theta_{*}$.

We check this independency in type A only. The idea is to identify the graded translation functors with the action of the $f_{i}$ 's on the Fock space; thus our combinatorics is really the combinatorics that calculates the global crystal basis. Since the global crystal basis is unique, the singular combinatorics will have no ambiguity either.

Let us now therefore briefly review the correspondence between Young diagrams and weights in type $\mathrm{A}_{n}$.

Let $\lambda_{i}$ be the length of the $i$ 'th line of the Young diagram $Y(\lambda)$. Then $Y(\lambda)$ is associated with the weight

$$
\lambda=\left(\lambda_{1}-\lambda_{2}, \lambda_{2}-\lambda_{3}, \ldots, \lambda_{n-1}-\lambda_{n}, \lambda_{n}\right) \in P^{+}
$$

due to the fact that the simple root $\alpha_{i}$ in type $A_{n}$ has coordinates

$$
\alpha_{i}=\left(0,0, \ldots, \stackrel{\downarrow i}{1},-\stackrel{\downarrow i+1}{1}^{+i+1} 0,0, \ldots\right)
$$


We conclude that $\lambda+\rho$ lies on a wall corresponding to $\alpha_{i}$ iff the last residues of the $i$ 'th and the $i+1$ 'th rows are equal. This generalizes to other roots: if the last residues of two rows are equal, the weight will lie on a wall. If one ends up on a wall by removing one node from the upper of two lines, then the corresponding weight lies above the wall and so on.

Example 8. $(l=3)$

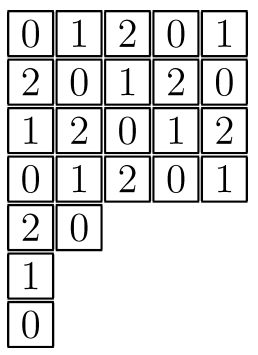

The 0 -residues give rise to three walls containing this $\lambda+\rho$. The removable 1 node means that $\lambda+\rho$ is positioned above two walls and below one wall coming from the 0 -nodes.

Let us now focus on the $n_{0}$ rows of $Y$ having as last node a 0 -node. Let $Y^{\prime}$ be the Young diagram obtained from $Y$ by adding one node to one of the rows (such a $Y^{\prime}$ may not exist).

We need to recall some facts on the modular representation theory of $G L_{m}(k)$.

Let $\Delta(\lambda)$ be the Weyl module given by $\lambda \vdash n$. It is a module for $G L_{m}(k)$ for any $m \geq n$. According to the branching rule $(\alpha=\lambda, \beta=1$ in (2.30) of [8]) we have the following identity in the Grothendieck group:

$$
\Delta(\lambda) \otimes E=\sum_{\substack{\operatorname{Young}(\lambda) \subseteq \operatorname{Young}(\mu) \\|\operatorname{Young}(\mu) \backslash \operatorname{Young}(\lambda)|=1}} \Delta(\mu)
$$

Here $E=\Delta(1)$, i.e. the natural module for $G L_{m}(k)$. Using Donkin's version of the Nakayama conjecture [4] we obtain:

$$
\text { (*) } p r_{\lambda^{\prime}}(\Delta(\lambda) \otimes E)=\sum_{\substack{\operatorname{Young}(\lambda) \subseteq \operatorname{Young}(\mu) \\ \mid \operatorname{Young}(\mu) \operatorname{Young}(\lambda)=\{1-\text { node }\}}} \Delta(\mu)
$$

Here $p r_{\lambda^{\prime}}$ denotes projection onto the block of $\Delta\left(\lambda^{\prime}\right)$; this is thus a formula for $T_{\lambda}^{\lambda^{\prime}}$ in the Grothendieck group.

We are now going to calculate a graded version of this formula, in other words we are going to apply the operators $\Theta_{*}$ and $\Theta^{*}$ of the singular combinatorics of the previous section.

Consider firstly the situation where the $n_{0}$ ' 0 '-nodes are all removable and assume furthermore that $n_{1}=0$; i.e. no row has a a ' 1 '-node at the end. 
Example 9. $(l=3)$

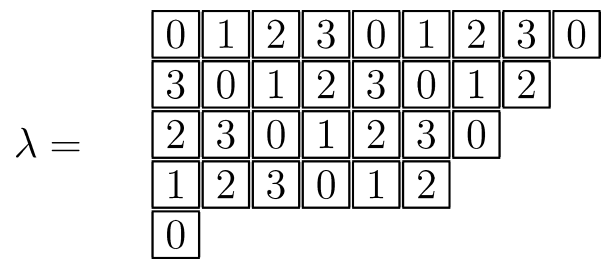

The $n_{0}$ ' 0 '-nodes give rise to $\left(\begin{array}{c}n_{0} \\ 2\end{array}\right)$ hyperplanes through $\lambda$. The other residues also give rise to hyperplanes through $\lambda$; on the other hand, the components of $T_{\lambda}^{\lambda^{\prime}} \Delta(\lambda)$ all stay fixed with respect to these other hyperplanes since we are adding only ' 1 '-nodes in $(*)$.

The components of $T_{\lambda}^{\lambda^{\prime}} \Delta(\lambda)$ lie on fewer hyperplanes than $\lambda$ since $n_{1}=0$ and thus we are in position to apply $\Theta_{\lambda, \lambda^{\prime}}^{*}$ from our singular combinatorics.

We must for each component $\Delta(\mu)$ of $T_{\lambda}^{\lambda^{\prime}} \Delta(\lambda)$ calculate the number $u\left(\mu, \Gamma_{\lambda}\right)=$ $\#\{$ hyperplanes $H$ through $\lambda$ with $\mu<H\}$ With the above $\lambda$, one of the occurring $\mu$ 's will be

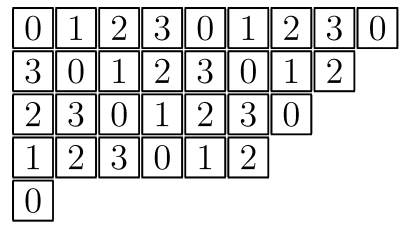

$\lambda$

\begin{tabular}{|c|c|c|c|c|c|c|c|c|}
\hline 0 & 1 & \begin{tabular}{|l||}
2 \\
\end{tabular} & 3 & 0 & 1 & \begin{tabular}{|l|}
2 \\
\end{tabular} & 3 & 0 \\
\hline 3 & 0 & \begin{tabular}{|l|}
1 \\
\end{tabular} & \begin{tabular}{|l|}
2 \\
\end{tabular} & 3 & 0 & 1 & 2 & \\
\hline 2 & 3 & 0 & \begin{tabular}{|l|}
1 \\
\end{tabular} & 2 & 3 & 0 & & \\
\hline 1 & 2 & 3 & 0 & 1 & 2 & & & \\
\hline 0 & 1 & & & & & & & \\
\hline
\end{tabular}

$\mu$

One sees in this example (which is easily generalized to all $\mu$ ) that

$$
u\left(\mu, \Gamma_{\lambda}\right)=\#\{0 \text {-nodes of } \lambda \text { above } \gamma\}
$$

where $\gamma=\mu \backslash \lambda$. Thus $\Delta(\mu)$ occurs in the graded translation from $\lambda$ to $\lambda^{\prime}$ with a shift of order:

$$
\begin{aligned}
& -u\left(\mu, \Gamma_{\lambda}\right)=-\#\{0 \text {-nodes of } \lambda \text { above } \gamma\} \\
& \quad=-\#\{\text { indent } 1 \text {-nodes of } \lambda \text { above } \gamma\}+\#\{\text { removable 1-nodes of } \lambda \text { above } \gamma\}
\end{aligned}
$$

We now consider the slightly more general situation where some of the ' 0 '-nodes of $\lambda$ are allowed to be non-removable; on the other hand we will require that there be no removable ' 1 '-nodes in $\lambda$. One then gets that

$$
n_{1}=\#\{\text { non-removable } 0 \text {-nodes of } \lambda\}
$$

Example 10. $(l=3)$

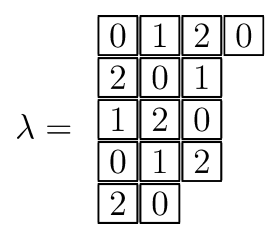

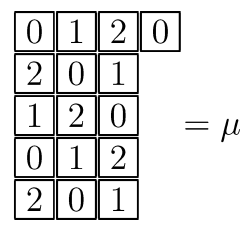


The $n_{0}$ ' 0 '-nodes still give rise to $\left(\begin{array}{c}n_{0} \\ 2\end{array}\right)$ walls passing through $\lambda$, but in this situation $\Theta_{\lambda, \lambda \text { ' }}^{*}$ is not a graded version of $T_{\lambda}^{\lambda^{\prime}}$, since $\lambda^{\prime}$ lies on walls not passing through $\lambda$.

The correct graded version of $T_{\lambda}^{\lambda^{\prime}}$ will be $\Theta_{*}^{\tau, \lambda^{\prime}} \circ \Theta_{\lambda, \tau}^{*}$, where $\tau \in X(T) \otimes \mathbb{R}$ is more regular than each of the weights $\lambda$ and $\lambda^{\prime}$. In our example $\tau$ can be visualized by the following "diagram".

$$
\tau=\begin{array}{|l||l|l||l|}
\hline 0 & 1 & 2 & 0 \\
\hline \hline 2 & 0 & 1 & \\
\hline \hline 1 & 2 & 0 \\
\hline \hline 0 & 1 & 2 \\
\hline \hline 2 & 0 & & \\
\cline { 1 - 2 } & &
\end{array}
$$

where we have added "half a node".

We then have

$$
u\left(\tau, \Gamma_{\lambda}\right)=\#\{0 \text {-nodes of } \lambda \text { above } \mu \backslash \lambda\}
$$

Furthermore we have that

$$
o\left(\tau, \Gamma_{\mu}\right)=\#\{1 \text {-nodes of } \lambda \text { above } \mu \backslash \lambda\}
$$

So $\Delta(\mu)$ appears in the graded translation functor from $\lambda$ to $\lambda^{\prime}$ with a shift of size

$$
\begin{aligned}
& -u\left(\tau, \Gamma_{\lambda}\right)+o\left(\tau, \Gamma_{\mu}\right)=-\#\{\text { indent } 1 \text {-nodes of } \lambda \text { above } \mu \backslash \lambda\} \\
& =-\#\{\text { indent } 1 \text {-nodes of } \lambda \text { above } \mu \backslash \lambda\}+\#\{\text { removable 1-nodes of } \lambda \text { above } \mu \backslash \lambda\}
\end{aligned}
$$

Consider finally the general situation in which we allow removable ' 1 '-nodes.

Our graded version of $T_{\lambda}^{\lambda^{\prime}}$ will then behave like $\oplus \Theta_{*}^{\tau, \lambda^{\prime}} \circ \Theta_{\lambda, \tau}^{*}$ where $\tau \in X(T) \otimes \mathbb{R}$ is chosen as before. Each $\Delta(\mu)$ appearing in the graded translation corresponds to a $\tau$ and the same calculation as before gives a shift of size

$$
\begin{aligned}
& \text {-\#\{ indent } 1 \text {-nodes of } \lambda \text { above } \mu \backslash \lambda\} \\
& +\#\{\text { removable } 1 \text {-nodes of } \lambda \text { above } \mu \backslash \lambda\}
\end{aligned}
$$

Hence we get in all cases exactly the number $-N_{1}^{r}(\lambda, \mu)$ of LLT. We can of course repeat this argument for the other residues and we conclude that the $f_{i}$ operators on the Fock space are really those graded translation functors in our singular setup that are summands of the tensor product with the natural module. Let us formulate this as a theorem:

Theorem 1. Let $f_{i}$ be one of the standard generators of $U_{q}\left(\widehat{\mathfrak{s l}}_{l}\right)$ and let $f_{i} \lambda=\sum c_{\mu}(q) \mu$ in the action of $f_{i}$ on $\mathcal{F}_{q}$. Then $\sum c_{\mu}(q) \mu$ equals $\Theta \Delta(\lambda)$ in the singular combinatorics of the previous section, where $\Theta$ is the operator of the singular combinatorics corresponding to $\lambda$ and $\mu$, with $\mu$ being obtained from $\lambda$ by adding an $i$-node. 
Now recall the selfdual element $w_{\lambda}$ of $\mathcal{F}_{q}$, which is the first step of the LLT-algorithm. It is on the form

$$
w_{\lambda}=f_{i_{1}}^{\left(n_{1}\right)} f_{i_{2}}^{\left(n_{2}\right)} \ldots f_{i_{k}}^{\left(n_{k}\right)} \emptyset
$$

for some $i_{k}$ and $n_{k}$ where $f_{i}^{(n)}=\left([n]_{q} !\right)^{-1} f_{i}^{n}$ is the usual divided power notation.

We therefore need to check that also the action of the higher divided powers $f_{i}^{(n)}=$ $\left([n]_{q} !\right)^{-1} f_{i}^{n}$ can be described in the singular combinatorics. This is an argument close to the above. Let us start with an example.

Example 11. $(l=3)$

$\tau=$\begin{tabular}{|l||l|l||l||l|l||l||l|l|}
\hline 0 & 1 & 2 & 3 & 0 & 1 & 2 & 3 & 0 \\
\hline \hline 3 & 0 & 1 & 2 & 3 & 0 & & & \\
\hline \hline 2 & 3 & 0 & & &
\end{tabular}

It corresponds to a Steinberg weight in the $A_{2}$ situation. Translating to the weight given by the diagram $\sigma$ :

$$
\sigma=\begin{array}{|l||l||l||l|l|l|l|l||l|l|}
\hline 0 & 1 & 2 & 3 & 0 & 1 & 2 & 3 & 0 & 1 \\
\hline \hline 3 & 0 & 1 & 2 & 3 & 0 & 1 & & & \\
\hline \hline 2 & 3 & 0 & & & &
\end{array}
$$

can be described in our combinatorics by the following picture.

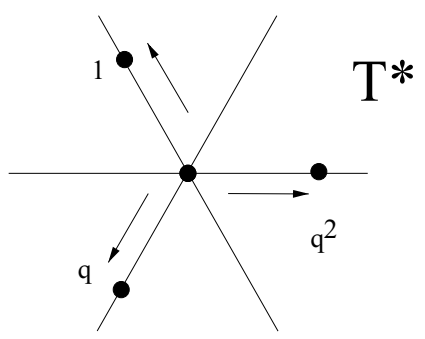

This is performed in the LLT-algorithm as a two step operation, adding one node in each step. In the alcove geometry we get the following picture

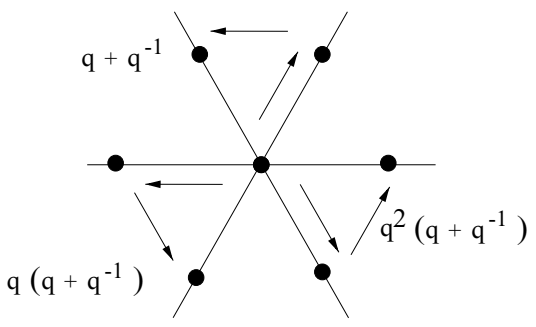

and so $\Theta^{*}$ corresponds to $f_{1}^{(2)}=\frac{1}{[2]} f_{1}^{2}$. 
The example generalizes to all $n$ :

Theorem 2. The action of $f_{i}^{(n)}$ on $\mathcal{F}_{q}$ corresponds to a $\Theta$ of the singular combinatorics.

Proof: We may assume that $i \equiv 1 \bmod l$. Assume first that the residues of the end nodes of $\lambda$ are all different from 1 . Then the end nodes of residue 0 all give rise to addable 1 -nodes. Let us consider $f_{1}^{(n)} \lambda$. Let $I=\left\{i_{l_{1}}, i_{l_{2}}, \ldots, i_{l_{K}}\right\}$ be the lines of $\lambda$ having 0 as end residue and let $\lambda^{J}$ for $J \subset\left\{l_{1}, \ldots, l_{K}\right\}$ with $|J|=n$ be the partition obtained from $\lambda$ by adding one node to the lines of $i_{l_{j}}$ for $j \in J$. The pairs $(a, b)$ of line numbers where $a \in I$ such that $a<j \forall j \in J$ and $b \in J$ correspond to the hyperplanes through $\lambda$ that lie above $\lambda^{J}$, i.e. those contributing to $-u\left(\lambda^{J}, \Pi\right)$ of Definition 1 where $\lambda \in \Pi$. But in the notation of Lemma 6.2 of [9] there are exactly $N(i d)+\left(\begin{array}{c}n \\ 2\end{array}\right)$ of these (where $n=k_{s}$ in loc. cit.). Since this is also the coefficient of $\lambda^{J}$ in $f_{1}^{(n)} \lambda$ the proof is finished in that case.

If there are 1-nodes occurring at the end of some lines, the situation is slightly more complicated since the relevant operator in the combinatorics is a composite of $\Theta^{*}$ and $\Theta_{*}$. Let $J$ be as before. Let us first assume that these 1-nodes are all removable and let us denote by $K$ the corresponding line numbers. Then the pairs $(a, b)$ of line numbers where $a \in K$ with $a<j \forall j \in J$ and $b \in J$ gives the number of hyperplanes through $\lambda$ lying below $\lambda^{J}$, i.e. contributing to $o\left(\lambda^{J}, \Pi\right)$. By Definition 1 we should subtract this number from $-u\left(\lambda^{J}, \Pi\right)$ and thus find once more the exact correspondence with the formula for $f_{1}^{(n)} \lambda$.

Actually, this argument also holds in the case where some of these 1-nodes are nonremovable, by our definition of $\Theta$ via "half"-nodes and we are done.

We can now prove that the singular combinatorics is well defined:

Theorem 3. Let the root system be of type A. Then the singular combinatorics is well defined, i.e. does not depend on the path of weights.

Proof: Let $\mu^{1}, \mu^{1}, \ldots, \mu^{k}, \ldots, \mu^{N}$ with $\mu^{1}$ in the fundamental alcove $C$ and finishing with $\mu^{N}=\lambda$. We view the weights as partitions and show that each of the operators $\Theta_{\mu^{i}, \mu^{i+1}}$ of the singular combinatorics applied to $\mu^{i}$ can be identified with the action of $f_{i_{1}}^{\left(n_{1}\right)} f_{i_{2}}^{\left(n_{2}\right)} \cdots f_{i_{p}}^{\left(n_{p}\right)}$ on $v^{i} \in \mathcal{F}_{q}$ for some choice of $f_{i_{j}}$ and $n_{i_{j}}$. But then the uniqueness of the crystal basis of $M \subset \mathcal{F}_{q}$ shows that the crystal basis is independent of the choice of path.

Let $T \subset G=S l_{m}$ be a maximal torus so that $\mu^{k}$ defines a $T$-module. We associate a partition $\left(\mu_{1}^{i}, \mu_{2}^{i}, \ldots, \mu_{m}^{i}\right)$ with each of the $\mu^{i}$ by the rule $\left\langle\mu^{i}, \alpha_{1}\right\rangle=\mu_{1}^{i}-\mu_{2}^{i}$ etc. This partition is unique up to adding the first columns a number of times.

As usual, we let $\rho$ denote the half sum of the positive roots, the corresponding partition being $(m, m-1, \ldots, 1)$. Recall that $l$ is the order of the root of unity. Then the position of $\mu^{i}$ in the alcove geometry with respect to $l$ is equal to the position of $r \mu^{i}+(r-1) \rho$ with respect to the alcove geometry defined by $r l$. Indeed letting $\omega_{i}$ denote the $i$ 'th fundamental weight we have that

$$
\left\langle r \lambda+(r-1) \rho+\rho, \omega_{i}\right\rangle=r\left\langle\lambda+\rho, \omega_{i}\right\rangle
$$

and hence the end residues of $r \lambda+(r-1) \rho$ equal the $r$-multiples of the residues of $\lambda$. Thus increasing the size of $r$ gives rise to more weights inside the alcoves and makes it possible to "separate" hyperplanes through $\lambda$ coming from different residues.

包 Springer 
We choose $r$ large enough for all of the operators $\Theta$ on the weights $\mu^{i}$ to be on the form $\Theta^{*}$ or $\Theta_{*}$, that is involving no composites of such. Furthermore, we obtain by choosing $r$ big enough that all the occurring weights $\mu^{i}$ are $l$-regular partitions.

Write $\mu^{i}=\sigma$ and $\mu^{i+1}=\tau$ and let us first assume that the operator that takes $\sigma$ to $\tau$ is of the type $\Theta_{*}$. We check that it can realized through a sequence of $f_{i}^{(k)}$,s.

By assumption we have that $\tau_{i}-\tau_{j}-i+j \equiv 0 \bmod l \Rightarrow \sigma_{i}-\sigma_{j}-i+j \equiv 0 \bmod l$. Let $I_{0} \subset[1, \ldots, m]$ consist of those indices $i$ such that $\tau_{i}-i \equiv 0 \bmod l$ and let similarly $J_{0}$ be those indices $i$ such that $\sigma_{i}-i \equiv 0 \bmod l$. Then $I_{0}$ determines the hyperplanes passing through $\tau$ that come from the residue $0 \bmod l$ and similarly $J_{0}$. Thus by assumption we have $I_{0} \subset J_{0}$.

Let $j \in I_{0} \backslash J_{0}$ be minimal. We can now add nodes to the $j$ 'th line of $\sigma$ until the last residue becomes 0 . Each node added on the way does not give rise to any new coinciding residues, since otherwise there would be a hyperplane separating $\sigma$ and $\tau$. Similarly we deal with the other elements of $\in I_{0} \backslash J_{0}$. But adding such nodes corresponds to the operation of $f_{i}$ where $i$ is the residue of the node. The other elements of $j \in I_{0} \backslash J_{0}$ are dealt with similarly.

At this stage, $\sigma$ and $\tau$ are in the same facette and we can add or subtract nodes to $\sigma$, without producing coinciding residues, to arrive at $\tau$. Adding these nodes corresponds to the operation of certain $f_{i}$ 's while subtracting of nodes corresponds to certain $e_{i}$ 's. But using the relations of $U_{q}\left(\widehat{\mathfrak{s l}}_{l}\right)$, these cancel out and we are done in this case.

Assume now that the operator that takes $\mu^{i}$ to $\mu^{i+1}$ is of the type $\Theta^{*}$ and write $\mu^{i}=\tau$ and $\mu^{i+1}=\sigma$. Thus, there is a root $\alpha$ such that $\langle\tau+\rho, \alpha\rangle \equiv 0$ while $\langle\sigma+\rho, \alpha\rangle \not \equiv 0$. We may assume that $\alpha$ is the only such root, by otherwise passing to a larger $l$. Write $\alpha=\omega_{k}-\omega_{l}$ for $k<l$ and assume wlog. that the residues of the $k^{\prime}$ th and $l$ 'th line of $\tau$ are 0 . Let $I_{0}$ be as before, i.e. $I_{0}$ defines the hyperplanes passing through $\tau$ coming from the residue 0 . Then the end residues of the lines in $\sigma$ of indices $I_{0}^{1}:=\left\{i \in I_{0} \mid i \leq k\right\}$ are constant and so are the end residues of the lines in $\sigma$ of indices $I_{0}^{2}:=\left\{i \in I_{0} \mid i \geq l\right\}$. Let the first constant be $n_{1}$ and the second be $n_{2}$. Assume first that $n_{1}=1$ and $n_{2}=0$. Then using Lemma 6.2 of [9] one checks that $f_{1}^{\left(\left|I_{0}^{1}\right|\right)}$ takes $\tau$ to $\sigma$.

For larger values of $n_{1}$ we instead operate with the composite $f_{n_{1}}^{\left(\left|I_{0}^{1}\right|\right)} \cdots f_{2}^{\left(\left|I_{0}^{1}\right|\right)} f_{1}^{\left(\left|I_{0}^{1}\right|\right)}$ on $\tau$ and for larger values of $n_{2}$ we first operates with $f_{n_{2}}^{\left(\left|I_{0}\right|\right)} \cdots f_{2}^{\left(\left|I_{0}\right|\right)} f_{1}^{\left(\left|I_{0}\right|\right)}$ on $\tau$ and then with a sequence of the first type.

This finishes the proof of the Theorem.

Remark . The paper [7] by Goodman and Wenzl contains a path algorithm for affine KazhdanLusztig polynomials valid for all Lie types. This gives a different proof of the Theorem.

Using the argument from the beginning of Section 5 we may now conclude that the LLTalgorithm calculates $[T(\lambda), \Delta(\mu)]$ for $\lambda$ a regular partition. But by a $q$-analogue of Karin Erdmann's result in [5], this number is equal to the decomposition number $d_{\lambda \mu}$ for Hecke algebras at an l'th root of unity. In other words, as claimed in the introduction of our paper, the LLT-conjecture follows from Soergel's algorithm.

Remark. The LLT conjecture only treats canonical basis coefficients $d_{\lambda, \mu}(q)$ for $\lambda$ an $l$ regular partition. On the other hand, the singular combinatorics defined in the present paper should work for arbitrary $\lambda$ as well and produce decomposition numbers for the $q$-Schur algebra. In [11], canonical basis coefficients $d_{\lambda, \mu}(q)$ were defined for arbitrary $\lambda$ and it was conjectured that their values at 1 coincide with these decomposition numbers. This conjecure was proved in [14]. 


\section{References}

1. H.H. Andersen, "Tensor products of quantized tilting modules," Commun. Math. Phys. 149 (1992), 149159.

2. S. Ariki, "On the decomposition numbers of the Hecke algebra $G(m, 1, n)$," J. Math. Kyoto Univ. 36 (1996) 789-808.

3. H.H. Andersen, J.C. Jantzen and W. Soergel, "Representations of quantum groups at a $p$-th root of unity and of semisimple Groups in characteristic $p$ : Independence of $p$," Astérisque 220 (1994).

4. S. Donkin, "On Schur algebras and related algebras. IV. The blocks of the Schur algebras," J. Algebra 168(2), (1994) 400-429.

5. K. Erdmann, "Symmetric groups and quasi-hereditary algebras. Finite dimensional algebras and related topics," in V. Dlab and L.L. Scott, (eds.), Kluwer, 1994, pp. 123-161.

6. F. Goodman and H. Wenzl, "Crystal basis of quantum affine algebras and affine Kazhdan-Lusztig polynomials," Internat. Math. Res. Notices 5 (1999), 251-275 MR1675980 (2000b:17017)

7. F. Goodman and H. Wenzl, "A path algorithm for affine Kazhdan-Lusztig polynomial," Math. Z. 237(2) (2001) 235-249. MR1838309 (2002i:20006)

8. G. James, "The decomposition matrices of $G l_{n}(q)$ for $n \leq 10$," Proc. London Math. Soc., 60 (1990) 225-265.

9. A. Lascoux, B. Leclerc, and J.-Y. Thibon, "Hecke algebras at roots of unity and crystal bases of quantum affine algebras," Commun. Math. Phys. 181 (1996) 205-263.

10. B. Leclerc and J.-Y. Thibon, "Littlewood-Richardson coefficients and Kazhdan-Lusztig polynomials, Combinatorial Methods in Representation Theory," Advanced Studies in Pure Mathematics 28 (2000) 155-220.

11. B. Leclerc, J.-Y. Thibon, "Canonical bases of $q$-deformed Fock spaces," Int. Math. Res. Notices, 9 (1996) $447-456$.

12. W. Soergel and Kazhdan-Lusztig-Polynome und eine Kombinatorik für Kipp-Moduln, Represent. Theory 1 (1997) 37-68.

13. W. Soergel, "Characterformeln für Kipp-Moduln über Kac-Moody Algebren, Representation Theory," 1 (1997) $115-132$.

14. M. Varagnolo and E. Vasserot, On the decomposition numbers of the quantized Schur algebra, Duke Math. J. 100(2) (1999) 267-297, MR1722955 (2001c:17029) 